\title{
Shikonin reduces TGF- $\beta 1$-induced collagen production and contraction in hypertrophic scar-derived human skin fibroblasts
}

\author{
CHEN FAN $^{1}$, YING DONG ${ }^{2}$, YAN XIE $^{1,3}$, YONGHUA SU $^{4}$, XUFANG ZHANG ${ }^{1}$, \\ DAVID LEAVESLEY ${ }^{1}$ and ZEE UPTON ${ }^{1,5}$
}

\author{
${ }^{1}$ Tissue Repair and Regeneration Program, Institute of Health and Biomedical Innovation, \\ Queensland University of Technology, Brisbane, Queensland $4059 ;{ }^{2}$ Cancer Research Program, \\ Translational Research Institute, Queensland University of Technology, Brisbane, Queensland 4102, Australia; \\ ${ }^{3}$ Tissue Organ Bank and Tissue Engineering Centre, General Hospital of Ningxia Medical University, Yinchuan, \\ Ningxia 750004; ${ }^{4}$ Department of Traditional Chinese Medicine, Changhai Hospital, Second Military Medical University, \\ Shanghai 200433, P.R. China; ${ }^{5}$ Wound Management Innovation Cooperative Research Centre,
} Brisbane, Queensland 4101, Australia

Received November 26, 2014; Accepted June 23, 2015

DOI: $10.3892 /$ ijmm.2015.2299

\begin{abstract}
Hypertrophic scarring/hypertrophic scars (HS) is a highly prevalent condition following burns and trauma wounds. Numerous studies have demonstrated that transforming growth factor- $\beta 1$ (TGF- $\beta 1$ ) plays an essential role in the wound healing process by regulating cell differentiation, collagen production and extracellular matrix degradation. The increased expression of TGF- $\beta 1$ is believed to result in the formation of HS. Shikonin (SHI), an active component extracted from the Chinese herb, Radix Arnebiae, has previously been found to downregulate the expression of TGF- $\beta 1$ in keratinocyte/fibroblast co-culture conditioned medium. In view of this, in this study, we aimed to further investigate the effects of SHI on TGF- $\beta 1$-stimulated hypertrophic scar-derived human skin fibroblasts (HSFs) and examined the underlying mechanisms. Cell viability and proliferation were measured using alamarBlue
\end{abstract}

Correspondence to: Chen Fan, Tissue Repair and Regeneration Program, Institute of Health and Biomedical Innovation, Queensland University of Technology, 60 Musk Avenue, Kelvin Grove, Queensland 4059, Australia

E-mail: c3.fan@connect.qut.edu.au

Abbreviations: $\alpha$ SMA, $\alpha$-smooth muscle actin; COL1A1, collagen, type I, alpha 1; COL3A1, collagen, type III, alpha 1; DMEM, Dulbecco's modified Eagle's medium; DMSO, dimethyl sulfoxide; ECM, extracellular matrix; ERK, extracellular signal-regulated kinase; FCS, fetal calf serum; HS, hypertrophic scarring; HSFs, hypertrophic scarderived human skin fibroblasts; MAPK, mitogen-activated protein kinase; RT-qPCR, reverse transcriptase-quantitative polymerase chain reaction; SDS-PAGE, sodium dodecyl sulphate polyacrylamide gel electrophoresis; SMAD2, SMAD family member 2; SHI, shikonin; TGF- $\beta 1$, transforming growth factor- $\beta 1$

Key words: shikonin, transforming growth factor- $\beta 1$, hypetrophic scar, fibroblasts, collagen and CyQUANT assays. The total amount of collagen and cell contraction were examined using Sirius red staining and the cell contraction assay kit. Gene expression and signalling pathway activation were detected using reverse transcription-quantitative polymerase chain reaction and western blot analysis. Our results revealed that SHI reduced TGF- $\beta 1$-induced collagen production through the ERK/Smad signalling pathway and attenuated TGF- $\beta 1$-induced cell contraction by downregulating $\alpha$-smooth muscle actin ( $\alpha$ SMA) expression in the HSFs. The data from this study provide evidence supporting the potential use of SHI as a novel treatment for HS.

\section{Introduction}

Hypertrophic scarring/hypertrophic scars (HS) is a frequent complication following burns and trauma wounds (1). Patients with HS often complain of pain, pruritus and loss of joint mobility (2). Although the pathological mechanisms underpinning HS formation remain elusive, it has been demonstrated that HS formation results from the dysregulation of the wound healing process (3). For example, exaggerated inflammation (4), the overabundant production of collagen (5), excessive contraction at the wound site (6), the reduced apoptosis of fibroblasts (7) or delayed re-epithelialization (8) during wound healing has been reported to lead to the formation of HS.

Transforming growth factor- $\beta 1$ (TGF- $\beta 1$ ) has been widely reported to play an essential role in wound healing and HS formation (9). In particular, there is evidence indicating that TGF- $\beta 1$ mediates fibroblast proliferation, collagen production, extracellular matrix (ECM) deposition and myofibroblast differentiation in the wound healing process (10). Moreover, it has been reported that the enhanced expression of TGF- $\beta 1$ stimulates collagen synthesis in fibroblasts by activating the Smad2/3 signalling pathways, resulting in the overabundant accumulation of collagen (11). In addition, the number of myofibroblasts, which decreases at the end of the normal wound healing process, continues to be found at high levels in 
HS tissues (12). The overexpression of TGF- $\beta 1$ has been shown to contribute to the persistence of myofibroblasts, since TGF- $\beta 1$ stimulates normal dermal fibroblasts to differentiate into myofibroblasts by upregulating $\alpha$-smooth muscle actin ( $\alpha$-SMA) expression (13). Therefore, TGF- $\beta 1$ is a key target for the development of novel therapeutic strategies for HS (14).

Shikonin ( $\mathrm{SHI}, \mathrm{C}_{16} \mathrm{H}_{16} \mathrm{O}_{5}$ ), an active component extracted from the Chinese herb, Radix Arnebiae, has been widely demonstrated to possess various biological activities, such as anti-inflammatory, anti-bacterial, anti-angiogenic and anti-tumorigenic properties (15). Importantly, in our preliminary studies, we found that SHI attenuated the expression of TGF- $\beta 1$ in keratinocyte/fibroblast co-culture conditioned medium, indicating the potential use of SHI as a treatment for HS [Fan et al (unpublished data)]. In this study, we aimed to investigate the effects of SHI on TGF- $\beta 1$-stimulated hypertrophic scar-derived human skin fibroblasts (HSFs) and examined the underlying mechanisms.

\section{Materials and methods}

Preparation of TGF- $\beta 1$ and SHI. TGF- $\beta 1$ (Merck, Sydney, NSW, Australia) was dissolved in $4 \mathrm{mM}$ hydrogen chloride $(\mathrm{HCl})$ containing $0.1 \%$ bovine serum albumin (BSA) (both from Sigma-Aldrich, Castle Hill, NSWales, Australia) and stored at $-20^{\circ} \mathrm{C}$ until use. SHI powder was produced by the National Institute for the Control of Pharmaceutical and Biological Products (Beijing, China). SHI was dissolved in dimethyl sulfoxide (DMSO; Sigma-Aldrich) as a stock solution and stored at $-20^{\circ} \mathrm{C}$ until use.

Cell culture. The HSFs isolated from 3 diffident patients were purchased from Cell Research Corp. (Singapore), with ethical approval obtained from the Queensland University of Technology (1300000063). The cells were cultured in Dulbecco's modified Eagle's medium (DMEM; Life Technologies, Victoria, Australia) containing $10 \%$ fetal calf serum (FCS; HyClone, Smithfield, QLD, Australia), $1 \% \mathrm{v} / \mathrm{v}$ penicillin/streptomycin solution and $1 \% \mathrm{v} / \mathrm{v} 2 \mathrm{mM}$ L-glutamine (both from Life Technologies) at $37^{\circ} \mathrm{C}$ in an incubator with $5 \% \mathrm{CO}_{2}$. The cell culture medium was replaced every 2-3 days.

Cell viability and proliferation. The effects of SHI on the viability and proliferation of the TGF- $\beta 1$-stimulated HSFs were determined using alamarBlue ${ }^{\circledR}$ and CyQUANT ${ }^{\circledR}$ assays, as previously described (16). Briefly, the HSFs $\left(6 \times 10^{4}\right.$ cells/ well) were seeded into 12 -well cell plates for $24 \mathrm{~h}$. Serial dilutions of SHI in medium containing $5 \mathrm{ng} / \mathrm{ml}$ TGF- $\beta 1$ were applied to the plates at final concentrations of $0,0.5$ and $1 \mu \mathrm{g} /$ ml. Treatments excluding TGF- $\beta 1$ and SHI were also included as controls. After $72 \mathrm{~h}$ of incubation, alamarBlue $(0.1 \mu \mathrm{M}$; Sigma-Aldrich) was added to each well and the plates were incubated at $37^{\circ} \mathrm{C}$ for a further $1 \mathrm{~h}$. The fluorescence in the samples from the wells was measured at $\lambda$ ex $570-10 \mathrm{~nm}$ and $\lambda$ em $590 \mathrm{~nm}$ using a POLARstar OPTIMA Microplate Reader (BMG Labtech, Ortenberg, Germany). To measure cell proliferation, the CyQUANT reagents (Life Technologies) were applied as per the manufacturer's instructions and cell proliferation was measured at $\lambda$ ex $485 \mathrm{P}$ and $\lambda$ em $520 \mathrm{P}$ using a POLARstar OPTIMA Microplate Reader (BMG Labtech).
Measurement of total collagen production. The total amount of collagen present in the HSF cultures was examined by Sirius red staining, as previously described (17). The HSFs were treated as described above. After $72 \mathrm{~h}$ of treatment, the cells were stained with Sirius red (Sigma-Aldrich) and incubated at $37^{\circ} \mathrm{C}$ for $90 \mathrm{~min}$. The plates were then washed with tap water and air-dried overnight. The Sirius red stain was dissolved in $0.1 \mathrm{M}$ sodium hydroxide $(\mathrm{NaOH}$; Sigma-Aldrich) and the absorbance was read at $540 \mathrm{~nm}$ using a 96-well microplate reader (Bio-Rad, Hercules, CA, USA).

Cell contraction assay. The effects of SHI on TGF- $\beta 1-$ stimulated HSF contraction were determined using a Cell Contraction assay kit (Jomar Bioscience, Adelaide South Australia), as per the manufacturer's instructions. Briefly, the HSFs were resuspended in medium containing various concentrations of SHI $(0,0.5$ and $1 \mu \mathrm{g} / \mathrm{ml})$ at $2 \times 10^{6}$ cells $/ \mathrm{ml}$. Subsequently, a $0.1 \mathrm{ml}$ cell suspension mixed with $0.4 \mathrm{ml}$ collagen solution provided by the assay kit was seeded into each well in a 24 -well plate at $37^{\circ} \mathrm{C}$ for $1 \mathrm{~h}$. Following collagen polymerization, $1 \mathrm{ml}$ DMEM was added on top of the collagen gels. The gels were incubated for 2 days, and were then detached from the plate. TGF- $\beta 1(5 \mathrm{ng} / \mathrm{ml})$ was added on the top of each gel and images of each gel were captured using a Nikon SMZ800 microscope (Nikon, Tokyo, Japan). The diameter of each gel was measured using a ruler at 0,12 , 24 and $48 \mathrm{~h}$.

Reverse trascription-quantitative polymerase chain reaction $(R T-q P C R)$. RT-qPCR was used to investigate gene expression in the HSFs treated with TGF- $\beta 1$ and SHI. The genes of interest and their respective primers are listed in Table I. The HSFs were treated as described above for 24 and $48 \mathrm{~h}$. Total RNA was then extracted from the cells using the Qiagen RNeasy Mini kit (Qiagen, Limburg, The Netherlands), as per the manufacturer's instructions. The RNA concentration was measured using a NanoDrop ${ }^{\circledR}$ ND-1000 Spectrophotometer (NanoDrop, Wilmington, DE, USA). First-strand cDNA synthesis was performed using Superscript ${ }^{\mathrm{TM}}$ III Reverse Transcriptase (Life Technologies). Briefly, the RNA sample (100 ng) from each group was mixed with $1 \mu \mathrm{l}$ random hexamers, $1 \mu \mathrm{l}$ deoxyribonucleotide triphosphate (dNTP) and DEPC-treated water to yield a final volume at $10 \mu \mathrm{l}$. The mixture was incubated at $65^{\circ} \mathrm{C}$ for $5 \mathrm{~min}$. Each sample was then further mixed with $2 \mu 1$ 10X RT buffer, $4 \mu \mathrm{l}$ magnesium chloride $\left(\mathrm{MgCl}_{2}\right), 2 \mu \mathrm{l}$ dithiothreitol (DTT), $1 \mu \mathrm{l}$ RNaseOUT and $1 \mu \mathrm{l}$ SuperScript RT and incubated at $25^{\circ} \mathrm{C}$ for $10 \mathrm{~min}, 50^{\circ} \mathrm{C}$ for $50 \mathrm{~min}$ followed by $5 \mathrm{~min}$ at $85^{\circ} \mathrm{C}$. Each sample was finally mixed with $1 \mu \mathrm{l}$ RNase $\mathrm{H}$ and incubated at $37^{\circ} \mathrm{C}$ for $20 \mathrm{~min}$. RT-qPCR was performed using the SYBR-Green reagent and analysed using the $\Delta \Delta \mathrm{Ct}$ method on an ABI 7500 Thermal Cycler (both from Life Technologies). The reaction for each group contained $10 \mu \mathrm{l}$ SYBR-Green reagent, $1 \mu \mathrm{M}$ forward and reverse primers and $100 \mathrm{ng}$ cDNA samples. Amplification was initiated at $95^{\circ} \mathrm{C}$ for $10 \mathrm{~min}$ followed by 40 cycles at $95^{\circ} \mathrm{C}$ for $15 \mathrm{sec}$ and finally terminated at $60^{\circ} \mathrm{C}$ for $1 \mathrm{~min}$. The cycle threshold $(\mathrm{Ct})$ value of the gene of interest was obtained and first normalised to the housekeeping gene glyceraldehyde 3-phosphate dehydrogenase (GAPDH) $(\triangle \mathrm{Ct})$ and then further converted to the control $(\Delta \Delta \mathrm{Ct})$. The fold-change of each target gene was calculated using the $2(-\Delta \Delta \mathrm{Ct})$ method. 
Table I. Primers used in RT-qPCR.

\begin{tabular}{lcl}
\hline Protein name & Corresponding gene name & Primer sequences \\
\hline Collagen I & COL1A1 & F: 5'-ACGAAGACATCCCACCAATC-3' \\
Collagen III & COL3A1 & R: 5'-AGATCACGTCATCGCACAAC-3' \\
aSMA & & F: 5'-GCCTCCCGGAAGTCAAGGAGAAAG-3' \\
& $\alpha M A$ & R: 5'-CTTTAGGACCGGGGAAGCCCATG-3' \\
Smad2 & & F: 5'-CTGCTGAGCGTGAGATTGTC-3' \\
& & R: 5'-CTCAAGGGAGGATGAGGATG-3' \\
GAPDH & GAPDH & F: 5'-GGCGAATCGGCGGGG-3' \\
& & R: 5'-CCTCTTGTATCGAACCTCCCG-3' \\
& & F: 5'-TCTTTTGCGTCGCCAGCCGAG-3'
\end{tabular}

F, forward; R, reverse; COL1A1, collagen, type I, alpha 1; COL3A1, collagen, type III, alpha 1; $\alpha$ SMA, $\alpha$-smooth muscle actin; GAPDH, glyceraldehyde 3-phosphate dehydrogenase; SMAD2, SMAD family member 2.

Identification of activated signalling pathways. The signalling pathways activated in the HSFs treated with SHI and stimulated with TGF- $\beta 1$ were determined by western blot analysis. The HSFs were treated as described above for 30 and $60 \mathrm{~min}$. Whole cell lysates were then collected in lysis buffer (RIPA buffer; SigmaAldrich) containing protease inhibitor cocktail (Roche Applied Science, Indianapolis, IN, USA), $2 \mathrm{mM}$ sodium vanadate and $10 \mathrm{mM}$ sodium fluoride (both from Sigma-Aldrich). The protein concentrations were measured using the bicinchoninic acid assay (BCA; Thermo Fisher Scientific, Waltham, MA, USA). Equal amounts of protein $(10 \mu \mathrm{g})$ from each cell lysate were prepared and separated using $12 \%$ sodium dodecyl sulphate polyacrylamide gel electrophoresis (SDS-PAGE) and then transferred onto nitrocellulose membranes (Bio-Rad). The membranes were incubated with primary antibodies overnight at $4^{\circ} \mathrm{C}$ in Odyssey blocking buffer (LI-COR Biosciences, Lincoln, NE, USA). Primary antibodies included extracellular signal-regulated kinase (ERK; \#9102, rabbit anti-human), phosphorylated ERK (p-ERK, \#9106, mouse anti-human) and Smad2/3 (\#3102, rabbit anti-human) from Genesearch (Arundel, QLD, Australia); phosphorylated Smad2/3 (p-Smad2/3, \#sc-11769-R, rabbit anti-human) from Santa Cruz Biotechnology, Inc., Santa Cruz, CA, USA; $\alpha-S M A$ (\#ab5694, rabbit anti-human) from Abcam (Cambridge, UK) and GAPDH (\#G8795, mouse anti-human) from Sigma-Aldrich. Secondary antibodies conjugated with Alexa Fluor 680 or 800 (Life Technologies) were then applied as species appropriate. Images were captured and analysed using the Odyssey Infrared Imaging system and software (LI-COR Biosciences).

Statistical analysis. All experiments were performed in triplicate, with each treatment tested individually 3 times in each assay using cells from 3 different patients. The data are expressed as the percentage of the control group. One-way ANOVA and Tukey's post-hoc test were applied and a value of $\mathrm{p}<0.05$ was considered to indicate a statistically significant difference.

\section{Results}

Effects of SHI on HSF viability and proliferation. To determine the effects of SHI on the viability and proliferation of TGF- $\beta 1$ - stimulated HSFs, the HSFs were treated with TGF- $\beta 1$ and various concentrations of SHI $(0,0.5$ and $1 \mu \mathrm{g} / \mathrm{ml})$. TGF- $\beta 1$ alone significantly increased the viability of the HSFs by $9.9 \pm 1.3 \%$ compared to the control (no treatment; $\mathrm{p}<0.05$; Fig. 1A). However, no effect of TGF- $\beta 1$ alone on HSF proliferation was observed (Fig. 1B). In addition, $\mathrm{SHI}$ at $0.5 \mu \mathrm{g} / \mathrm{ml}$ had no significant effect on the viability and proliferation of the TGF- $\beta 1$-stimulated HSFs, while SHI at $1 \mu \mathrm{g} / \mathrm{ml}$ decreased the viability and proliferation of the TGF- $\beta 1$-stimulated HSFs compared to the control $(\mathrm{p}<0.05)$ by $2.3 \pm 0.6$ and $7.7 \pm 1.5 \%$, respectively (Fig. 1). These data indicate that SHI reduces the TGF- $\beta 1$-induced increase in HSF viability in a dose-dependent manner.

SHI attenuates TGF- $\beta 1$-induced collagen production in $H S F s$. To determine the effects of SHI on collagen production in the TGF- $\beta 1$-stimulated HSFs, total collagen present in the HSFs was stained with Sirius red (Fig. 2A) and was then further analysed quantitatively (Fig. 2B). A greater amount of collagen (red staining) was observed in the HSFs treated with TGF- $\beta 1$ alone compared to the the control group (no treatment; Fig. 2A). However, decreased amounts of collagen were detected in the TGF- $\beta 1$-stimulated HSFs treated with SHI at 0.5 and $1 \mu \mathrm{g} / \mathrm{ml}$ (Fig. 2A). Consistent with the results from Sirius red staining, the quantitative data indicated that that TGF- $\beta 1$ alone significantly increased the total amount of collagen by $31.4 \pm 1.5 \%$ compared to the control group $(\mathrm{p}<0.05$; Fig. 2B). However, treatment with SHI at 0.5 and $1 \mu \mathrm{g} / \mathrm{ml}$ attenuated collagen production in the TGF- $\beta 1$-stimulated HSFs by $9.7 \pm 0.7$ and $27.4 \pm 1.9 \%$, respectively, compared to the group treated with TGF- $\beta 1$ alone $(\mathrm{p}<0.05$; Fig. $2 \mathrm{~B})$. These results demonstrate that SHI inhibits the TGF- $\beta 1$-induced increase in collagen production in HSFs in a dose-dependent manner.

TGF- $\beta 1$-induced contraction of HSFs is inhibited by SHI. The effects of SHI on HSF-mediated gel contraction were evaluated using a collagen gel contraction assay. Treatment with TGF- $\beta 1$ alone significantly enhanced the contraction of the gels containing the HSFs by $7.7 \pm 2.2,28.4 \pm 1.4$ and $40.3 \pm 5.0 \%$ at 12 , 24 and $48 \mathrm{~h}$, respectively, compared to the control group (no treatment; $p<0.05$; Fig. 3 ). In addition, the contraction of the 
A

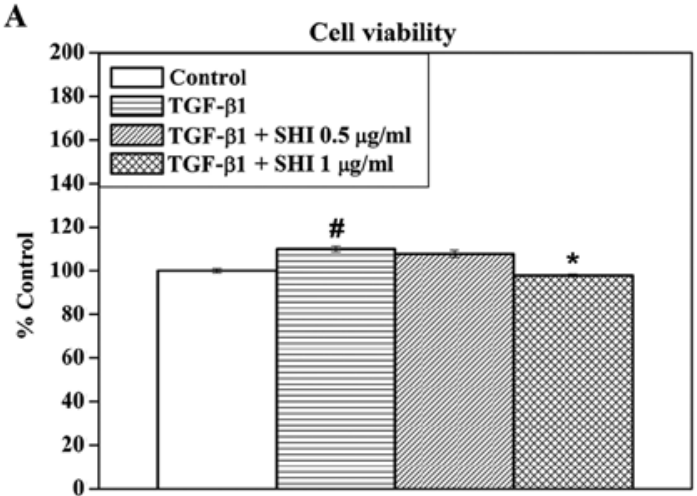

B

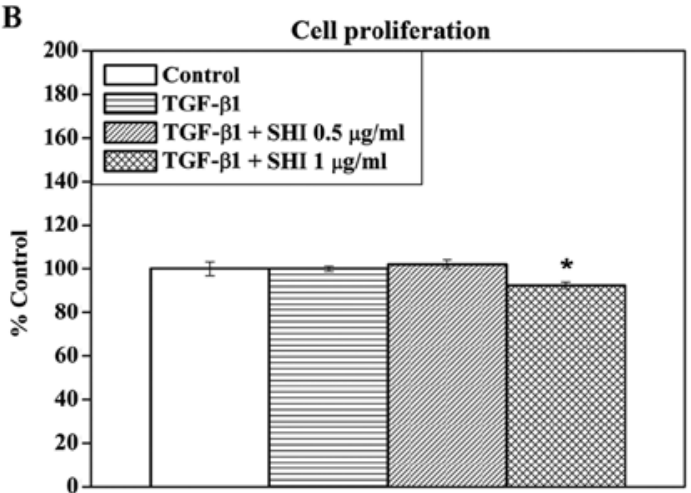

Figure 1. Effect of shikonin (SHI) on (A) cell viability and (B) cell proliferation. Hypertrophic scar-derived human skin fibroblasts (HSFs) were treated with transforming growth factor- $\beta 1$ (TGF- $\beta 1)$ and various concentrations of SHI $(0,0.5$ and $1 \mu \mathrm{g} / \mathrm{ml})$ for $72 \mathrm{~h}$. Culture medium containing neither TGF- $\beta 1 \mathrm{nor}$ SHI was used as a control treatment. Cell viability was measured using the alamarBlue assay, while cell proliferation was measured using the CyQUANT assay. The data are expressed as the average percentage of the control and were pooled from 3 replicate experiments (with cells from 3 different patients). Error bars indicate the mean \pm SEM ( $\mathrm{n}=3$ ). ${ }^{\#} \mathrm{p}<0.05$ vs. the control group; ${ }^{*} \mathrm{p}<0.05$ vs. the TGF- $\beta 1$ group. Statistical analysis was performed using one-way ANOVA and Tukey's post-hoc test.

A

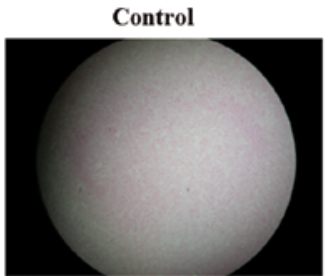

TGF- $\beta 1+$ SHI $0.5 \mu \mathrm{g} / \mathrm{ml}$

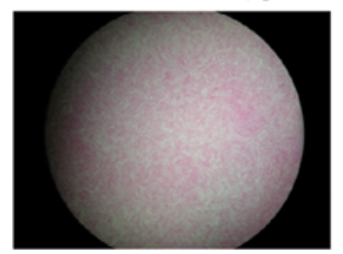

TGF- $\beta 1$

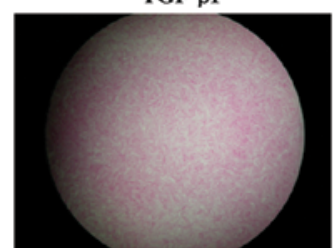

TGF- $\beta 1+$ SHI $1 \mu \mathrm{g} / \mathrm{ml}$

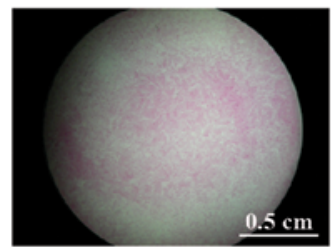

B

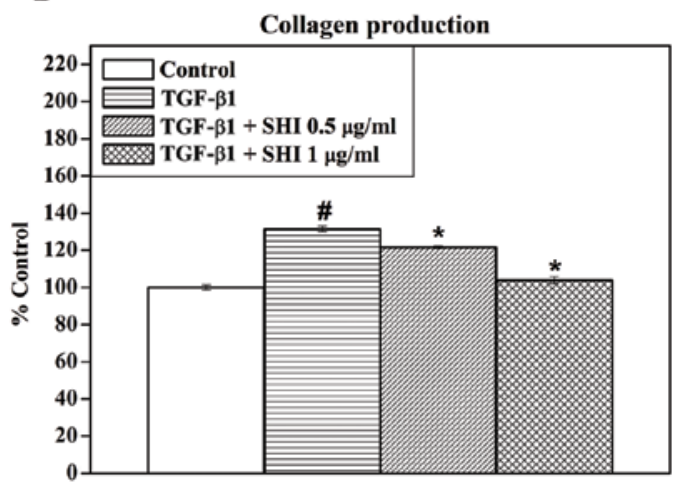

Figure 2. Effect of shikonin (SHI) on collagen production in transforming growth factor- $\beta 1$ (TGF- $\beta 1$ )-stimulated hypertrophic scar-derived human skin fibroblasts (HSFs). (A) Sirius red staining of collagen in HSF cultures. (B) Quantitative analysis of Sirius red staining. HSFs were treated with TGF- $\beta 1$ and various concentrations of SHI $(0,0.5$ and $1 \mu \mathrm{g} / \mathrm{ml})$ for $72 \mathrm{~h}$, and were then stained with Sirius red. Images were captured using a Nikon SMZ800 microscope. Quantitative data are expressed as the average percentage of the control and were pooled from 3 replicate experiments (with cells from 3 different patients). Error bars indicate the mean \pm SEM $(n=3) .{ }^{\#} \mathrm{p}<0.05$ vs. the control group (no TGF- $\beta 1$ or SHI treatment); ${ }^{*}<<0.05$ vs. the TGF- $\beta 1$ group. Statistical analysis was performed using one-way ANOVA and Tukey's post-hoc test.

gels containing HSFs treated with TGF- $\beta 1$ and $0.5 \mu \mathrm{g} / \mathrm{ml} \mathrm{SHI}$ was $14.1 \pm 1.3,31.3 \pm 2.3$ and $37.5 \pm 2.4 \%$ greater than that of the control group at 12, 24 and 48 h, respectively (p<0.05; Fig. 3). However, the HSFs treated with TGF- $\beta 1$ and $1 \mu \mathrm{g} / \mathrm{ml} \mathrm{SHI}$ showed no significant differences in gel contraction compared to the control group ( $p<0.05$; Fig. 3). Taken together, these results suggest that SHI attenuates the TGF- $\beta 1$-induced contraction of gels containing HSFs in a dose-dependent manner.

Effects of SHI on gene expression in TGF- $\beta 1$-stimulated HSFs. The gene expression of collagen, type I, alpha 1 (COL1A1), collagen, type III, alpha 1 (COL3A1), $\alpha-S M A$ and SMAD2 in the HSFs following treatment with TGF- $\beta 1$ and SHI was examined by RT-qPCR. Treatment with TGF- $\beta 1$ alone significantly upregulated COL1A1, $\alpha-S M A$ and SMAD2 gene expression in the HSFs at 24 and $48 \mathrm{~h}$ compared to the control group (no treatment; $p<0.05$; Fig. 4). No effect of TGF- $\beta 1$ on the expression of COL3A1 in the HSFs was observed (Fig. 4). However, treatment with SHI at either 0.5 or $1 \mu \mathrm{g} / \mathrm{ml}$ significantly decreased the expression of COL1A1, COL3A1, $\alpha-S M A$ and SMAD2 in the
HSFs compared to treatment with TGF- $\beta 1$ alone (Fig. 4), indicating that SHI suppresses the TGF- $\beta 1$-induced changes in gene expression in the HSFs.

SHI-induced activation of signalling pathways in HSFs. To elucidate the underlying mechanisms of SHI-induced decrease in collagen production in the TGF- $\beta 1$-stimulated HSFs, the expression of ERK, p-ERK, Smad2/3, p-Smad2/3 and $\alpha$-SMA was determined by western blot analysis. No effects of TGF- $\beta 1$ and SHI on the expression of ERK and Smad2/3 were detected in the HSFs (Fig. 5). However, the expression of p-ERK and p-Smad2/3 was significantly upregulated in the HSFs following treatment with TGF- $\beta 1$ alone for $30 \mathrm{~min}$, while the protein expression of these signalling molecules was decreased in the HSFs treated with both TGF- $\beta 1$ and SHI (either 0.5 or $1 \mu \mathrm{g} / \mathrm{ml}$ ) for 30 and $60 \mathrm{~min}$ (Fig. 5). In addition, the expression of $\alpha$-SMA increased in the HSFs treated with TGF- $\beta 1$ alone for 30 and $60 \mathrm{~min}$, whereas the TGF- $\beta 1$-induced upregulation of $\alpha$-SMA expression was inhibited when the HSFs were also treated with SHI (either 0.5 or $1 \mu \mathrm{g} / \mathrm{ml}$ ) for $60 \mathrm{~min}$. 
A
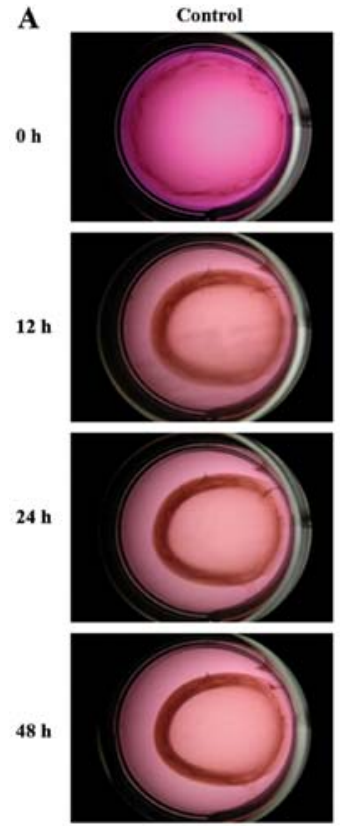

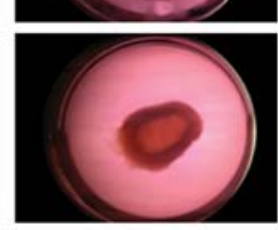

TGF- $\beta 1$
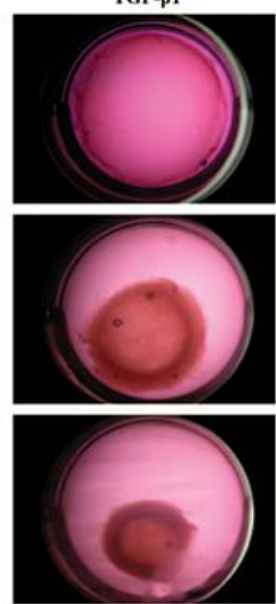

TGF- $\beta 1+$ SHI $0.5 \mu \mathrm{g} / \mathrm{ml}$
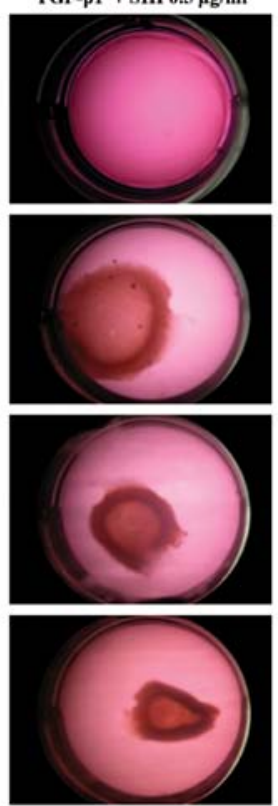

TGF- $\beta 1+$ SHI $1 \mu \mathrm{g} / \mathrm{ml}$
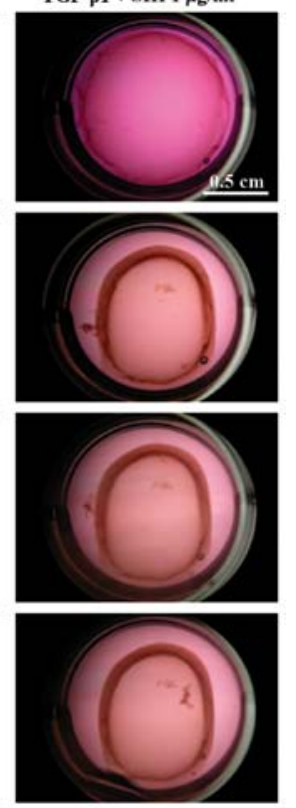

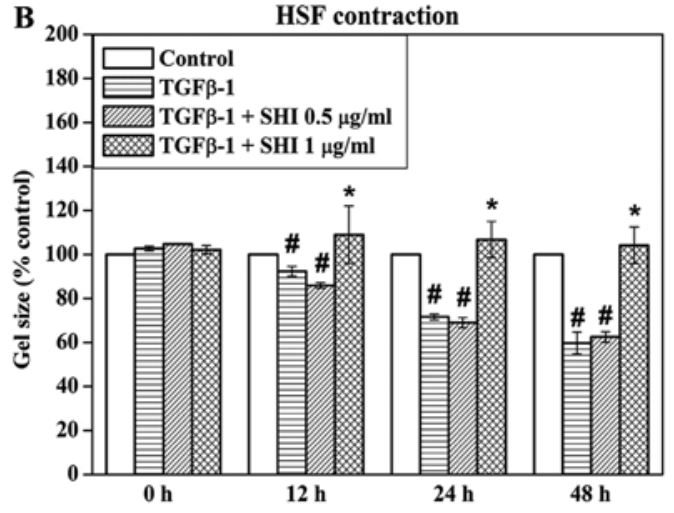

\section{Discussion}

TGF- $\beta 1$ is a multifunctional cytokine regulating cell proliferation, collagen production, cell differentiation and ECM degradation during wound healing processes (10). Enhanced levels of TGF- $\beta 1$ in cutaneous wound healing often leads to the formation of HS (11). Based on the data of our previous studies (unpublished data), in this study, we investigated the effects of SHI on TGF- $\beta 1$-stimulated HSFs and found that SHI reduced the TGF- $\beta 1$-induced collagen production in HSFs, as well as cell contraction, indicating the potential therapeutic value of SHI as a novel treatment strategy for HS.

During the early stages of wound healing, fibroblasts migrate to the wound lesion and produce collagen, assisting the replacement of the blood clot with new granulation tissue (18). Excessive collagen is degraded by proteases, such as collagenases, at the final stage of wound healing (19). TGF- $\beta 1$ has been demonstrated to play an essential role in the production of collagen by regulating the Smad2/3 signalling pathways (11). Following the binding of TGF- $\beta 1$ to its receptor, T $\beta$ R-I, the phosphorylation of Smad2/3 protein occurs intracellularly (20). p-Smad2/3 then heteromultimerises with Smad4, resulting in its transfer into the nucleus and the triggering of the expression of target genes, such as COLIAI (21). In accordance with the
Figure 3. Effect of shikonin (SHI) on gel contraction by transforming growth factor- $\beta 1$ (TGF- $\beta 1$ )-stimulated hypertrophic scar-derived human skin fibroblasts (HSFs). (A) Images of HSF contraction of collagen gels. (B) Quantitative analysis of HSF-mediated gel contraction. Cell contraction assay was performed following the manufacturer's instructions. Images were captured at $0,12,24$ and $48 \mathrm{~h}$ using a Nikon SMZ800 microscope. The cwere measured using a ruler and the gel size was calculated. Quantitative data are expressed as the average percentage of the control (no TGF- $\beta 1$ or SHI treatment) and were pooled from 3 replicate experiments (with cells from 3 different patients). Error bars indicate the mean $\pm \operatorname{SEM}(\mathrm{n}=3) .{ }^{*} \mathrm{p}<0.05$ vs. the control group; " $\mathrm{p}<0.05$ vs. the TGF- $\beta 1$ group. Statistical analysis was performed using one-way ANOVA and Tukey's post-hoc test.

results of previous studies (unpublished data), our data suggest that TGF- $\beta 1$ increases total collagen production, phosphorylates Smad2/3 and upregulates COL1A1 gene expression in HSFs. However, these changes induced by TGF- $\beta 1$ in the HSFs can be significantly attenuated when SHI treatment is concomitantly applied, indicating that SHI attenuates TGF- $\beta 1$-induced collagen production in HSFs.

TGF- $\beta 1$-induced Smad2/3 signalling has been reported to be associated with the activation of mitogen-activated protein kinases (MAPKs), including ERK, c-Jun N-terminal kinases (JNK) and mitogen-activated protein kinase 14 (also known as p38 $\alpha$ ) (22). The linker region of Smads, in particular, plays an essential role in mediating Smad function (23). Studies have indicated that ERK phosphorylates the linker region of $\mathrm{Smad} 2 / 3$, thereby regulating the function of $\mathrm{Smad} 2 / 3$ in fibroblasts $(24,25)$. Additionally, ERK inhibitors significantly block the phosphorylation of the Smad linker region (26). Furthermore, the activation of ERK has been observed to enhance the duration of Smad2/3 transcriptional activity (24). In order to provide insight into the underlying mechanisms responsible for the SHI-induced decrease in collagen production in the TGF- $\beta 1$-stimulated HSFs, we examined the expression of MAPKs following treatment with SHI. As shown by our results, the expression of phosphorylated JNK (p-JNK) and 

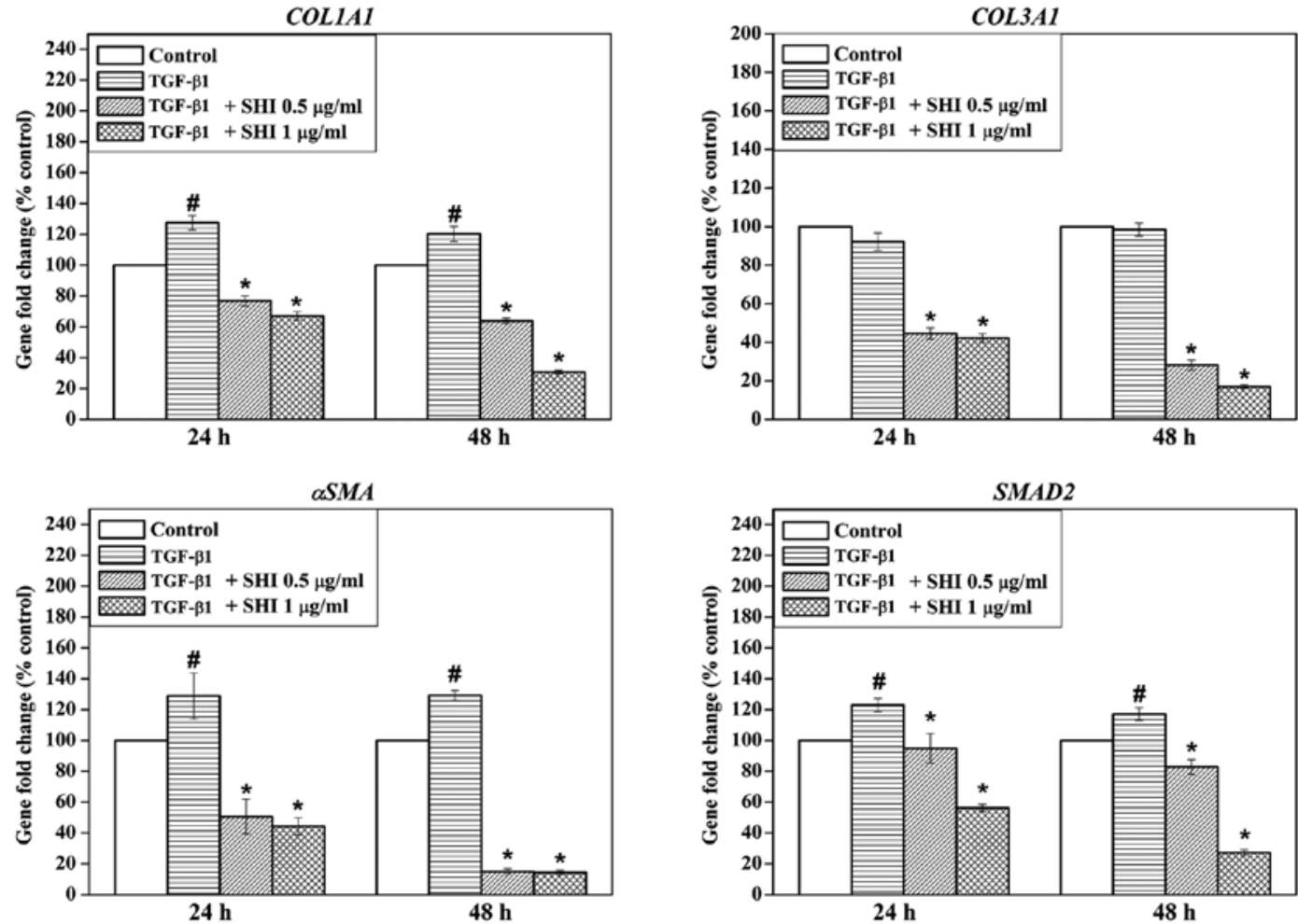

Figure 4. Effects of shikonin (SHI) on gene expression in transforming growth factor- $\beta 1$ (TGF- $\beta 1$ )-stimulated hypertrophic scar-derived human skin fibroblasts (HSFs). HSFs were treated with TGF- $\beta 1$ and various concentrations of SHI $(0,0.5$ and $1 \mu \mathrm{g} / \mathrm{ml})$ for 24 and $48 \mathrm{~h}$ and then total RNA was collected. Following RNA extraction, first-strand cDNA was synthesised. The cDNA sample was then amplified by RT-qPCR. The expression of the target genes was first normalised to glyceraldehyde 3-phosphate dehydrogenase (GAPDH) and then further converted to the percentage of the control (no TGF- $\beta 1$ or SHI treatment).

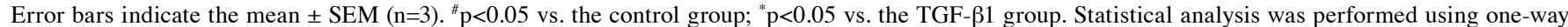
ANOVA and Tukey's post-hoc test.
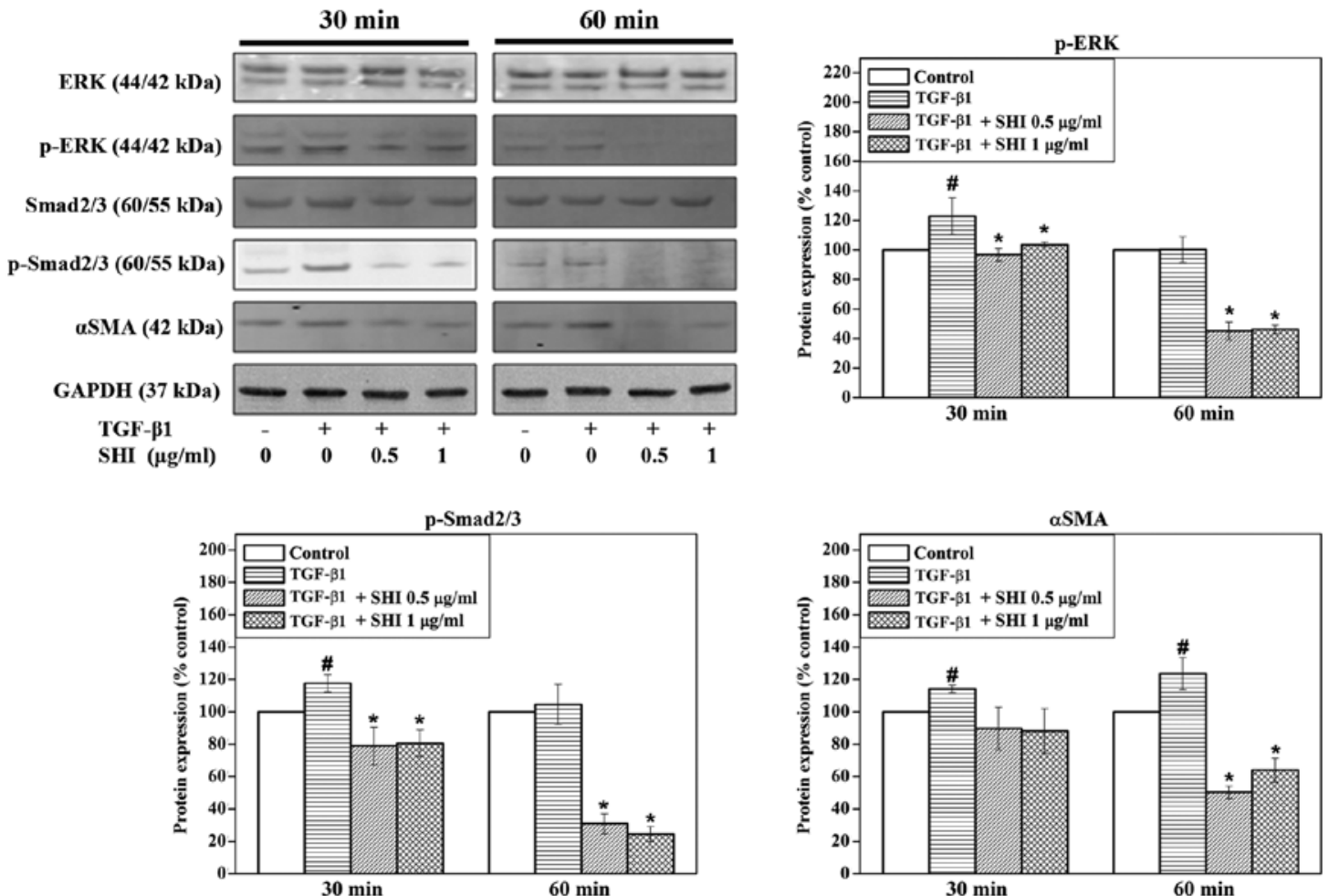

Figure 5. Shikonin (SHI)-induced signalling pathways in transforming growth factor- $\beta 1$ (TGF- $\beta 1$ )-stimulated hypertrophic scar-derived human skin fibroblasts (HSFs). HSFs were treated with TGF- $\beta 1$ and various concentrations of SHI $(0,0.5$ and $1 \mu \mathrm{g} / \mathrm{ml})$ for 30 and 60 min. The expression of target signalling proteins was detected using the Odyssey Infrared Imaging system. Glyceraldehyde 3-phosphate dehydrogenase (GAPDH) was included as a loading control. For quantitative analysis, the intensities of the bands were quantified by densitometry, normalised to GAPDH and then further converted to the percentage of the control (no TGF- $\beta 1$ or SHI treatment). Error bars indicate the mean \pm SEM $(n=3) .{ }^{~} p<0.05$ vs. the control group; ${ }^{*} p<0.05$ vs. the TGF- $\beta 1$ group. Statistical analysis was performed using one-way ANOVA and Tukey's post-hoc test. 
p38 (p-p38) was not evident in the HSFs following treatment with TGF- $\beta 1$ and SHI (data not shown). However, treatment with SHI significantly attenuated the TGF- $\beta 1$-induced phosphorylation of ERK (p-ERK) in the HSFs (Fig. 5). These data suggest that $\mathrm{SHI}$ reduces the TGF- $\beta 1$-induced collagen production in HSFs through the ERK/Smad signalling pathway.

During the formation of granulation tissue, some dermal fibroblasts change their phenotype to become myofibroblasts, which are responsible for wound contraction (27). Contraction is essential for the wound healing process as it reduces the wound size, thereby enabling wound closure. However, excessive wound contraction leads to the formation of HS (28). Myofibroblasts, cells characterised by the presence of $\alpha$-SMA (29), have been demonstrated to play a key role in HS formation (30). Indeed, an increased expression of $\alpha \mathrm{SMA}$ has been widely reported in HS tissues (31). TGF- $\beta 1$ has been shown to upregulate the expression of $\alpha \mathrm{SMA}$, as TGF- $\beta 1$ stimulates normal fibroblasts to differentiate into myofibroblasts (13). In this study, we also provide evidence that TGF- $\beta 1$ improves the ability of HSFs to contract gels and that it enhances the expression of $\alpha$ SMA in HSFs. Moreover, SHI attenuates the TGF- $\beta 1$-induced contraction of HSFs and $\alpha$ SMA upregulation in HSFs in a dose-dependent manner.

Taken together, the data reported herein indicate that TGF- $\beta 1$ plays a key role in cellular processes crucial to wound healing and HS formation. Increased levels of TGF- $\beta 1$ lead to the formation of HS by stimulating collagen production and wound contraction. Furthermore, to the best of our knowledge, this study demonstrates for the first time that SHI reduces TGF- $\beta 1$-induced collagen production through the ERK/ Smad signalling pathway and attenuates the TGF- $\beta 1$-induced contraction of gels containing HSFs by downregulating $\alpha \mathrm{SMA}$ expression. This evidence supports the potential use of SHI as a novel treatment strategy for HS.

\section{Acknowledgements}

The authors would like to acknowledge the support of the Australian Government's Cooperative Research Centres Program and the Queensland University of Technology (QUT) Tissue Repair and Regeneration Program. We also acknowledge the PhD scholarship from QUT and the Wound Management Innovation Cooperative Research Centre.

\section{References}

1. Aarabi S, Longaker MT and Gurtner GC: Hypertrophic scar formation following burns and trauma: New approaches to treatment. PLoS Med 4: e234, 2007.

2. Xiao Z,Zhang M, Liu Y and Ren L: Botulinum toxin type a inhibits connective tissue growth factor expression in fibroblasts derived from hypertrophic scar. Aesthetic Plast Surg 35: 802-807, 2011.

3. Sun Z, Li S, Cao C, Wu J, Ma B and Tran V: shRNA targeting SFRP2 promotes the apoptosis of hypertrophic scar fibroblast. Mol Cell Biochem 352: 25-33, 2011.

4. Liu XJ, Xu MJ, Fan ST, Wu Z, Li J, Yang XM, Wang YH, Xu J and Zhang ZG: Xiamenmycin attenuates hypertrophic scars by suppressing local inflammation and the effects of mechanical stress. J Invest Dermatol 133: 1351-1360, 2013.

5. Huang D, Shen KH and Wang HG: Pressure therapy upregulates matrix metalloproteinase expression and downregulates collagen expression in hypertrophic scar tissue. Chin Med J (Engl) 126: $3321-3324,2013$

6. Gauglitz GG, Korting HC, Pavicic T, Ruzicka T and Jeschke MG: Hypertrophic scarring and keloids: Pathomechanisms and current and emerging treatment strategies. Mol Med 17: 113-125, 2011.
7. Liu BH, Chen L, Li SR, Wang ZX and Cheng WG: Smac/DIABLO regulates the apoptosis of hypertrophic scar fibroblasts. Int J Mol Med 32: 615-622, 2013

8. Simon F, Bergeron D, Larochelle S, Lopez-Vallé CA, Genest H, Armour A and Moulin VJ: Enhanced secretion of TIMP-1 by human hypertrophic scar keratinocytes could contribute to fibrosis. Burns 38: 421-427, 2012.

9. Yin L, Zhao X, Ji S, He C, Wang G, Tang C, Gu S and Yin C: The use of gene activated matrix to mediate effective SMAD2 gene silencing against hypertrophic scar. Biomaterials 35: 2488-2498, 2014.

10. Liu S, Jiang L, Li H, Shi H, Luo H, Zhang Y, Yu C and Jin Y: Mesenchymal stem cells prevent hypertrophic scar formation via inflammatory regulation when undergoing apoptosis. J Invest Dermatol 134: 2648-2657, 2014.

11. Wang R, Ghahary A, Shen Q, Scott PG, Roy K and Tredget EE: Hypertrophic scar tissues and fibroblasts produce more transforming growth factor-betal mRNA and protein than normal skin and cells. Wound Repair Regen 8: 128-137, 2000.

12. Ehrlich HP, Desmoulière A, Diegelmann RF, Cohen IK, Compton CC, Garner WL, Kapanci Y and Gabbiani G: Morphological and immunochemical differences between keloid and hypertrophic scar. Am J Pathol 145: 105-113, 1994.

13. Varga $J$ and Abraham D: Systemic sclerosis: A prototypic multisystem fibrotic disorder. J Clin Invest 117: 557-567, 2007.

14. Colwell AS, Phan TT, Kong W, Longaker MT and Lorenz PH: Hypertrophic scar fibroblasts have increased connective tissue growth factor expression after transforming growth factor-beta stimulation. Plast Reconstr Surg 116: 1387-1392, 2005.

15. Chen X, Yang L, Oppenheim JJ and Howard MZ: Cellular pharmacology studies of shikonin derivatives. Phytother Res 16: 199-209, 2002

16. Jones LJ, Gray M, Yue ST, Haugland RP and Singer VL: Sensitive determination of cell number using the CyQUANT cell proliferation assay. J Immunol Methods 254: 85-98, 2001.

17. Malkusch W, Rehn B and Bruch J: Advantages of Sirius Red staining for quantitative morphometric collagen measurements in lungs. Exp Lung Res 21: 67-77, 1995.

18. Singer AJ and Clark RA: Cutaneous wound healing. N Engl J Med 341: 738-746, 1999.

19. Urioste SS, Arndt KA and Dover JS: Keloids and hypertrophic scars: Review and treatment strategies. Semin Cutan Med Surg 18: 159-171, 1999.

20. Piek E, Moustakas A, Kurisaki A, Heldin CH and ten Dijke P: TGF-(beta) type I receptor/ALK-5 and Smad proteins mediate epithelial to mesenchymal transdifferentiation in NMuMG breast epithelial cells. J Cell Sci 112: 4557-4568, 1999.

21. Wrana JL and Attisano L: The Smad pathway. Cytokine Growth Factor Rev 11: 5-13, 2000

22. Massague J: Integration of Smad and MAPK pathways: A link and a linker revisited. Genes Dev 17: 2993-2997, 2003.

23. Inman GJ: Linking Smads and transcriptional activation. Biochem J 386: e1-e3, 2005.

24. Hough C, Radu M and Doré JJ: Tgf-beta induced Erk phosphorylation of smad linker region regulates smad signaling. PLoS One 7: e42513, 2012.

25. Derynck R and Zhang YE: Smad-dependent and Smadindependent pathways in TGF-beta family signalling. Nature 425 : $577-584,2003$

26. Li J, Zhao Z, Liu J, Huang N, Long D, Wang J, Li X and Liu Y: MEK/ERK and $\mathrm{p} 38 \mathrm{MAPK}$ regulate chondrogenesis of rat bone marrow mesenchymal stem cells through delicate interaction with TGF-beta1/Smads pathway. Cell Prolif 43: 333-343, 2010.

27. Darby IA and Hewitson TD: Fibroblast differentiation in wound healing and fibrosis. Int Rev Cytol 257: 143-179, 2007.

28. Zhang Z, Garron TM, Li XJ, Liu Y, Zhang X, Li YY and Xu WS: Recombinant human decorin inhibits TGF- $\beta 1$-induced contraction of collagen lattice by hypertrophic scar fibroblasts. Burns 35: 527-537, 2009.

29. Franz M, Spiegel K, Umbreit C, Richter P, Codina-Canet C, Berndt A, Altendorf-Hofmann A, Koscielny S, Hyckel P, Kosmehl H, et al: Expression of Snail is associated with myofibroblast phenotype development in oral squamous cell carcinoma. Histochem Cell Biol 131: 651-660, 2009.

30. Ibrahim MM, Bond J, Bergeron A, Miller KJ, Ehanire T, Quiles C, Lorden ER, Medina MA, Fisher M, Klitzman B, et al: A novel immune competent murine hypertrophic scar contracture model: A tool to elucidate disease mechanism and develop new therapies. Wound Repair Regen 22: 755-764, 2014.

31. Wang J,Dodd C,Shankowsky HA, Scott PG and TredgetEE; Wound Healing Research Group: Deep dermal fibroblasts contribute to hypertrophic scarring. Lab Invest 88: 1278-1290, 2008. 